\section{Prevalência da hipertensão arterial, do diabetes mellitus e da adesão às medidas comportamentais no Município de São Paulo, Brasil, 2003-2015}

\section{Prevalence of arterial hypertension, diabetes mellitus, and adherence to behavioral measures in the city of São Paulo, Brazil, 2003-2015}

\section{Prevalencia de la hipertensión arterial, diabetes mellitus y adhesión a las medidas comportamentales en el municipio de São Paulo, Brasil, 2003-2015}

\author{
Sheila Rizzato Stopa 1 \\ Chester Luiz Galvão Cesar 1 \\ Neuber José Segri 2 \\ Maria Cecilia Goi Porto Alves 3 \\ Marilisa Berti de Azevedo Barros 4 \\ Moisés Goldbaum 5
}

doi: 10.1590/0102-311X00198717

\section{Resumo}

O objetivo foi comparar as estimativas de prevalência de diabetes, hipertensão e as medidas de controle para estas doenças. Foram analisados dados de população adulta provenientes dos Inquéritos de Saúde no Município de São Paulo, Brasil, 2003, 2008 e 2015. Foram estimadas as prevalências e seus intervalos de 95\% de confiança (IC95\%) para as variáveis: hipertensão, diabetes $e$ as práticas de controle para estas doenças (dieta alimentar, atividade física, medicamento oral, insulina, não faz nada). As estimativas foram comparadas por regressão de Poisson ajustada por sexo e idade, e analisadas segundo os domínios 20-59 e 60 anos e mais. Os dados foram apresentados comparando-se os anos de 2008 em relação a 2003 e 2015 em relação a 2003. Entre as pessoas de 20-59 anos, observou-se aumento nas prevalências de: hipertensão no período 2003-2015 ( $R P=1,27$; IC95\%: 1,03-1,60) e dieta alimentar para ambos os períodos (2003-2008, $R P=2,04$; IC95\%: 1,42-2,91; e 2003-2015, $R P=1,51$; IC95\%: 1,05-2,15). Dentre as pessoas com 60 anos e mais: diabetes $(R P=1,29$; IC95\%: 1,08-1,56) e medicamento oral para controlar a diabetes $(R P=1,38$; IC95\%: 1,17-1,63), ambos no período 2003-2015; hipertensão no período 2003-2015 ( $R P=1,19$; IC95\%: 1,05-1,39); e dieta alimentar e medicamento oral para controlar a hipertensão no periodo 2003-2008 (RP = 1,20; IC95\%: 0,95-1,51 e RP = 1,02; IC95\%: 0,95-1,09, respectivamente). Os resultados são importantes para a vigilância e monitoramento dos indicadores analisados, e fornecem subsídio ao planejamento de ações em saúde no Município de São Paulo. Articular e alinhar ações efetivas e integradas é imprescindivel para a redução e controle dessas doenças.

Hipertensão; Diabetes Mellitus; Doença Crônica; Monitoramento Epidemiológico; Inquéritos Epidemiológicos

\author{
Correspondência \\ S. R. Stopa \\ Programa de Pós-graduação em Saúde Pública, Faculdade de \\ Saúde Pública, Universidade de São Paulo. \\ Av. Dr. Arnaldo 715, São Paulo, SP 01246-904, Brasil. \\ sheilarstopa@gmail.com \\ 1 Faculdade de Saúde Pública, Universidade de São Paulo, São \\ Paulo, Brasil. \\ 2 Universidade Federal de Mato Grosso, Cuiabá, Brasil. \\ 3 Instituto de Saúde, São Paulo, Brasil. \\ 4 Faculdade de Ciências Médicas, Universidade Estadual de \\ Campinas, Campinas, Brasil. \\ 5 Faculdade de Medicina, Universidade de São Paulo, São Paulo, \\ Brasil.
}




\section{Introdução}

As doenças crônicas não transmissíveis (DCNT) constituem um dos maiores e mais desafiadores problemas de saúde pública e têm correspondido a um elevado número de mortes prematuras, perda de qualidade de vida e incapacidades para realizar atividades da vida diária, além do impacto econômico para as sociedades e sistemas de saúde 1,2,3.

Do total de mortes ocorridas no mundo em 2012, 38 milhões foram em decorrência das DCNT, das quais $3 / 4$ (28 milhões) ocorreram em países de baixa e média rendas 2 . No Brasil, elas foram responsáveis por mais de 74\% do total de mortes em 2014 4 . Dentre as DCNT mais prevalentes na população adulta e idosa, destaca-se a hipertensão arterial e o diabetes mellitus como corresponsáveis pelas principais causas de mortalidade e hospitalizações no país 5 .

Ainda no Brasil, de acordo com dados da Pesquisa Nacional de Saúde (PNS 2013), as prevalências de diabetes mellitus e hipertensão arterial foram de 6,2\% (intervalo de 95\% de confiança - IC95\%: 5,9-6,6) e 21,4\% (IC95\%: 20,8-22,0), respectivamente. Com relação aos dados do Município de São Paulo, mais especificamente, as prevalências foram de 8,4\% (IC95\%: 7,1-9,7) para diabetes e 21,0\% (IC95\%: 19,1-22,8) para hipertensão 6,7,8. Segundo dados do VIGITEL (Vigilância e Fatores de Risco e Proteção para Doenças Crônicas por Inquérito Telefônico), as prevalências de diabetes no Município de São Paulo passaram de 6,2\% (IC95\%: 5,1-7,3) em 2006 - no início de seu monitoramento - para 7,7\% (IC95\%: 6,4-9,1) em 2015 9,10.

A detecção precoce, tratamento e controle da hipertensão arterial e do diabetes mellitus são essenciais para a redução de seus agravos relacionados 11,12. Adotar medidas para a modificação de estilos de vida torna-se imprescindível para o efetivo controle dessas doenças. Manter uma alimentação saudável e praticar atividade física regularmente, agregadas - ou não - a tratamento medicamentoso, são exemplos de práticas que atuam no controle e terapêutica. Investimentos em diagnóstico precoce, manutenção da adesão ao tratamento designado e acesso à assistência de qualidade são medidas efetivas em saúde pública 2,13,14.

A possibilidade de monitorar as prevalências e medidas de controle para hipertensão arterial e diabetes mellitus em diferentes períodos permite conhecer a magnitude e tendência dessas doenças, bem como o perfil das medidas adotadas pela população, fundamentais no subsídio de planejamento de ações e definição de prioridades em saúde 15,16. O objetivo do presente estudo foi comparar as estimativas de prevalência de diabetes, hipertensão e as medidas de controle para estas doenças, utilizando dados do Inquérito de Saúde do Município de São Paulo (ISA-Capital) 2003, 2008 e 2015.

\section{Métodos}

Neste trabalho foram analisados dados de população adulta (20 anos e mais) provenientes do ISACapital, inquérito domiciliar de base populacional realizado no Município de São Paulo, nos anos de 2003, 2008 e 2015, apoiado pela Secretaria Municipal de Saúde de São Paulo por meio de convênio com o Centro de Apoio à Faculdade de Saúde Pública da Universidade de São Paulo.

A escolha da área temática foi feita pela importância socioeconômica do Município de São Paulo, o mais populoso do país, com cerca de 20 milhões de habitantes vivendo em sua região metropolitana, e com Índice de Desenvolvimento Humano (IDH) de 0,805. Apesar do IDH ser alto, a distribuição não é homogênea dentro do município, uma vez que distritos mais centrais apresentam IDH superior a 9,0 e diminui gradualmente à proporção que se afasta do centro da cidade 17. Dado o contexto socioeconômico do município e a vinculação das instituições de ensino com a Secretaria Municipal de Saúde de São Paulo, nas edições do ISA-Capital foram abordados temas relacionados às condições de vida e saúde dos moradores do Município de São Paulo, como estilo de vida, estado de saúde e utilização de serviços de saúde.

No ISA-Capital 2003 foram entrevistados 3.357 indivíduos e a amostragem foi estratificada por conglomerados em dois estágios: censitários e domicílios. Foram sorteados 60 setores com base na amostra da PNAD 2002 (Pesquisa Nacional por Amostra de Domicílios), agrupados em três estratos, segundo a escolaridade do chefe da família, estimada pelo percentual de chefes que possuíam nível universitário: $<5 \%, 5 \%$ a $24,99 \%$ e $\geq 25 \%$. Esse procedimento visou a aumentar a probabilidade de 
inclusão de indivíduos que pertenciam aos estratos de maior nível socioeconômico, assegurando a análise de indicadores de situação de saúde segundo grupos sociais distintos. O tamanho da amostra foi calculado considerando-se o cenário correspondente à máxima variabilidade para a frequência dos eventos estudados, com base na estimativa de prevalência de 50\%, nível de $95 \%$ de confiança, erro de amostragem de 0,06 e efeito do delineamento de 1,5.

No ISA-Capital 2008 foram entrevistados 3.271 indivíduos. A amostragem foi por conglomerados em dois estágios: setores censitários (70 setores) e domicílios, sem estratificação do município. A amostra foi calculada considerando-se a estimativa de prevalência de 50\%, nível de $95 \%$ de confiança, erro de amostragem de 0,04 a 0,07 e efeito do delineamento de 1,5.

Em ambos os anos, as amostras foram formadas por oito domínios demográficos: $<1$ ano; 1-11 anos; 12-19 anos (masculino e feminino); 20-59 anos (masculino e feminino); $\mathrm{e} \geq 60$ anos (masculino e feminino).

No ISA-Capital 2015 foram entrevistados 4.043 indivíduos. A amostragem foi estratificada por conglomerados em dois estágios: setores censitários e domicílios. O município foi estratificado pelas coordenadorias regionais de saúde: Norte, Centro-oeste, Sudeste, Sul e Leste, também consideradas como domínios de estudo. Foram sorteados 30 setores censitários em cada coordenadoria, baseando-se na listagem de setores urbanos do Censo Demográfico 2010. A amostra do ISA-Capital 2015 foi formada pelos domínios: 12-19 anos (masculino e feminino), 20-59 anos (masculino), 20-59 anos (feminino) e $\geq 60$ anos (masculino e feminino) e calculada com base em uma estimativa de prevalência de $50 \%$, com erro de amostragem de 0,10, considerando-se um nível de $95 \%$ de confiança e um efeito de delineamento de 1,5 .

Com relação às perdas, tanto em 2003 como em 2008, considerou-se um percentual de 20\% de perdas para corrigir os efeitos de não resposta. Já para o ISA-Capital 2015, esse percentual foi de $26 \%$. Maiores detalhes metodológicos sobre os planos de amostragem das edições do ISA-Capital podem ser acessados no site da pesquisa e em publicação oficial 18,19.

Para este estudo, consideraram-se os domínios amostrais de 20-59 anos, dos sexos masculino e feminino, e de 60 anos e mais, dos sexos masculino e feminino, provenientes dos três inquéritos utilizados, correspondendo a um total de 1.667 indivíduos para o ano de 2003, 2.086 em 2008 e 3.184 no ano de 2015, num total de 6.937 indivíduos.

Foram selecionadas para o estudo: variáveis socioeconômicas e demográficas: sexo, idade (em anos), raça/cor da pele autodeclarada (branca, preta e parda), ocupação (com atividade, sem atividade - aposentados, pensionistas e donas de casa -, desempregado, estudante), escolaridade em anos completos ( $<5,5-8$, 9 e mais), renda familiar per capita em salários mínimos $-(\leq 0,5 ;>0,5$ a $\leq 1 ;>1$ a $<2,5$; $\geq 2,5 \mathrm{SM}$ ); variáveis de saúde: presença de diabetes, presença de hipertensão, medidas autorreferidas de controle para estas doenças (dieta alimentar, prática de atividade física, uso de medicamento oral de rotina, uso de insulina de rotina - apenas para quem referiu diabetes -, não faz nada).

As variáveis socioeconômicas e demográficas foram utilizadas para caracterizar a população deste trabalho. As análises foram realizadas em um único banco de dados, no qual foram combinadas as informações dos três inquéritos para as variáveis estudadas. As variáveis foram renomeadas e seus valores e rótulos categorizados identicamente, e uma nova variável foi criada para identificar a origem da informação (ISA-Capital 2003, 2008 ou 2015), possibilitando comparações entre os inquéritos 20. Além disso, foram consideradas as diferenças de delineamento no processo de estratificação dos inquéritos utilizados.

Foram estimadas as prevalências e IC95\% para as variáveis selecionadas, categorizadas segundo os domínios de idade: 20-59 anos e 60 anos e mais. As razões de prevalência (RP) foram calculadas por regressão de Poisson com variância robusta, no intuito de estimar a magnitude das diferenças entre as estimativas encontradas nos inquéritos. As variáveis sexo e idade foram utilizadas como ajuste nos modelos de regressão para controlar possíveis discrepâncias entre as diferentes distribuições de frequência das amostras.

As características de interesse estudadas foram consideradas como variáveis dependentes e a variável criada para identificar a origem do inquérito (ISA-Capital 2003, 2008 ou 2015) como independente. Assim, se a variável independente fosse estatisticamente significativa, existiria evidência para considerar a presença de diferença entre os inquéritos utilizados, com nível descritivo de 0,05 para o teste de Wald. As RP foram apresentadas comparando-se os anos de 2008 em relação a 2003 e 2015 em relação a 2003. 
A análise dos dados foi realizada usando-se o programa estatístico Stata 14.0 (https://www.stata. com/), por meio do módulo survey, que considera os efeitos da amostragem complexa. Todos os indivíduos entrevistados foram consultados, esclarecidos e aceitaram participar da pesquisa. Este estudo foi aprovado pelo Comitê de Ética da Faculdade de Saúde Pública da Universidade de São Paulo (parecer no 1.368.925, de 2015).

\section{Resultados}

A caracterização da população de estudo segundo as variáveis demográficas e socioeconômicas pode ser observada na Tabela 1. Na análise da distribuição percentual dessas variáveis observou-se uma predominância de mulheres, pessoas de 20-39 anos de idade e que tinham 9 ou mais anos de estudos. Ainda, foram encontradas maiores proporções de adultos que se autodeclararam de raça/cor de pele branca e que exerciam algum tipo de atividade. Com relação à renda per capita em SM, maior proporção de pessoas apresentou renda > 2,5 SM no ano de 2003, e em 2015 esta proporção foi maior entre os que referiram renda $<0,5 \mathrm{SM}$.

\section{Tabela 1}

Distribuição percentual da população adulta (20 anos e mais), segundo características socioeconômicas e demográficas e ano de realização dos inquéritos. ISA-Capital 2003, 2008 e 2015, São Paulo, Brasil.

\begin{tabular}{|c|c|c|c|c|c|c|}
\hline \multirow[t]{2}{*}{ Características } & \multicolumn{2}{|c|}{ ISA-Capital 2003} & \multicolumn{2}{|c|}{ ISA-Capital 2008} & \multicolumn{2}{|c|}{ ISA-Capital 2015} \\
\hline & $n$ * & \% (IC95\%) ** & $n$ * & \% (IC95\%) ** & $\mathrm{n}$ * & $\%($ IC95\%) ** \\
\hline \multicolumn{7}{|l|}{ Sexo } \\
\hline Masculino & 803 & $45,1(42,1-48,2)$ & 848 & $46,3(44,2-48,4)$ & 1.340 & $46,3(44,5-48,0)$ \\
\hline Feminino & 864 & $54,9(51,8-58,0)$ & 1.238 & $53,7(51,6-55,8)$ & 1.844 & $53,7(52,0-55,5)$ \\
\hline \multicolumn{7}{|l|}{ Faixa etária (anos) } \\
\hline $20-39$ & 502 & $52,0(48,8-55,3)$ & 645 & $47,4(44,7-50,2)$ & 1.175 & $46,2(44,0-48,3)$ \\
\hline $40-59$ & 293 & $32,0(29,1-35,0)$ & 517 & $36,3(33,8-38,8)$ & 990 & $35,3(33,5-37,2)$ \\
\hline 60 e mais & 872 & $16,0(14,1-18,1)$ & 924 & $16,3(14,1-18,8)$ & 1.019 & $18,5(16,6-20,6)$ \\
\hline \multicolumn{7}{|l|}{ Raça } \\
\hline Branca & 1.077 & $68,6(64,7-72,2)$ & 1.311 & $63,3(59,2-67,3)$ & 1.630 & $55,3(51,6-59,0)$ \\
\hline Preta & 133 & $8,4(6,3-11,0)$ & 153 & $7,4(5,7-9,7)$ & 322 & $10,8(9,4-12,3)$ \\
\hline Parda & 373 & $23,0(19,6-26,9)$ & 574 & $29,2(25,7-33,0)$ & 1.015 & $33,9(30,8-37,3)$ \\
\hline \multicolumn{7}{|l|}{ Ocupação } \\
\hline Com atividade & 766 & $61,3(57,6-64,7)$ & 1.069 & $68,3(65,5-71,0)$ & 1.870 & $66,4(64,1-68,5)$ \\
\hline Sem atividade & 108 & $8,5(6,6-10,9)$ & 95 & $5,6(4,4-7,1)$ & 214 & $7,6(6,4-8,9)$ \\
\hline Desempregado & 728 & $26,3(23,6-29,1)$ & 883 & $23,5(21,1-26,1)$ & 1.019 & $23,3(21,4-25,4)$ \\
\hline Estudante & 47 & $4,0(2,6-5,9)$ & 38 & $2,7(1,8-3,9)$ & 71 & $2,7(2,1-3,6)$ \\
\hline \multicolumn{7}{|l|}{ Escolaridade (anos) } \\
\hline$<5$ & 601 & $23,0(20,6-25,7)$ & 636 & $16,0(13,9-18,3)$ & 743 & $17,2(15,5-19,0)$ \\
\hline $5-8$ & 267 & $20,7(18,2-23,3)$ & 399 & $18,6(16,0-21,5)$ & 554 & $16,2(14,6-18,0)$ \\
\hline 9 ou mais & 325 & $26,9(24,0-30,0)$ & 593 & $38,1(35,1-41,2)$ & 1.074 & $36,4(34,3-38,5)$ \\
\hline \multicolumn{7}{|c|}{ Renda familiar per capita (em SM) } \\
\hline$\leq 0,5$ & 354 & $19,8(16,8-23,1)$ & 436 & $18,6(15,2-22,7)$ & 1.062 & $33,2(29,2-37,4)$ \\
\hline$>0,5 a \leq 1$ & 385 & $20,4(16,9-24,3)$ & 520 & $22,1(18,3-26,5)$ & 748 & $21,9(19,4-24,5)$ \\
\hline$>1 \mathrm{a}<2,5$ & 522 & $29,8(26,3-33,6)$ & 763 & $35,8(32,2-39,6)$ & 898 & $27,9(25,2-30,9)$ \\
\hline$\geq 2,5$ & 406 & $30,1(25,2-35,4)$ & 367 & $23,4(19,5-27,9)$ & 476 & $17,0(14,5-19,9)$ \\
\hline
\end{tabular}

IC95\%: intervalo de 95\% de confiança; SM: salário mínimo.

* Número de indivíduos na amostra não ponderada;

** Prevalências e IC95\% calculados sob ponderação. 
Na Tabela 2, são apresentadas as prevalências e IC95\% para as variáveis de saúde selecionadas, referentes aos domínios de 20-59 anos de idade. Os dados sobre a diabetes para essa faixa de idade não foram apresentados em função de IC95\% muito amplos e coeficientes de variação maiores que 30\%. A prevalência de hipertensão autorreferida no ano de 2003 foi de 11,7\% (IC95\%: 9,4-14,5), passando para 16,0\% (IC95\%: 14,3-17,9) em 2015, com aumento de 27\% no período 2003-2015 (RP = 1,27; IC95\%: 1,03-1,60). A dieta alimentar para controle da hipertensão também apresentou aumento nos períodos analisados (2003-2008, RP = 2,04; IC95\%: 1,42-2,91 e 2003-2015, RP = 1,51; IC95\%: 1,05-2,15).

Os dados relativos aos domínios de 60 anos e mais, para as variáveis de saúde analisadas, são apresentados na Tabela 3. A prevalência de diabetes autorreferido nos indivíduos de 60 anos e mais também apresentou aumento significativo no período: em 2003 a prevalência estimada era de 17,6\% (IC95\%: 14,9-20,6), passando para 22,5\% (IC95\%: 20,0-25,2) no ano de 2015. Constatou-se, após ajuste, uma elevação de $29 \%$ na prevalência de diabetes no período 2003-2015 (RP = 1,29; IC95\%: 1,08-1,56). Com relação às medidas de controle para diabetes, a estimativa de prevalência do uso de medicamento oral de rotina para controlar a doença apresentou diferenças estatisticamente significativas nos dois períodos de análise: 2003-2008 RP = 1,19 (IC95\%: 1,00-1,40) e 2003-2015 RP = 1,38 (IC95\%: 1,17-1,63), respectivamente.

A prevalência de hipertensão autorreferida também apresentou aumento significativo no domínio analisado para o período 2003-2015: passou de 46,2\% (IC95\%: 41,5-51,5) no ano de 2003 para 54,9\% (IC95\%: 51,0-58,6) em 2015, expressando após ajuste um aumento de $19 \%$ no período $(\mathrm{RP}=1,19$; IC95\%: 1,05-1,39). Contatou-se, também, aumento no período 2003-2008 para as variáveis dieta alimentar ( RP = 1,57; IC95\%: 1,23-2,00) e medicamento oral (RP = 1,06; IC95\%: 1,00-1,13) (Tabela 3).

As demais medidas de controle para diabetes/hipertensão, de ambos os domínios de idade analisados, não apresentaram diferença estatisticamente significativa entre as estimativas dos três inquéritos.

\section{Discussão}

Neste estudo foram investigadas as prevalências de diabetes e hipertensão, bem como as práticas autorreferidas adotadas como forma de controle para estas doenças. As informações são oriundas de inquéritos de base populacional periódicos e possibilitaram o monitoramento de tais condições de saúde ao longo do tempo. Entender a distribuição, magnitude e tendência dessas doenças é, também, fortalecer a vigilância das DCNT, prioridade nacional 1,15,21,22.

\section{Tabela 2}

Prevalências e razões de prevalências ajustadas de hipertensão arterial e suas medidas de controle em população adulta (20-59 anos), segundo ano de realização dos inquéritos. ISA-Capital 2003, 2008 e 2015, São Paulo, Brasil.

\begin{tabular}{|c|c|c|c|c|c|c|c|c|c|c|}
\hline \multirow[t]{2}{*}{ Variáveis } & \multicolumn{2}{|c|}{$\begin{array}{c}\text { ISA-Capital } 2003 \\
(n=795) *\end{array}$} & \multicolumn{2}{|c|}{$\begin{array}{l}\text { ISA-Capital } 2008 \\
(n=1.162) *\end{array}$} & \multicolumn{2}{|c|}{$\begin{array}{c}\text { ISA-Capital } 2015 \\
(n=2.165) \text { * }\end{array}$} & \multirow[t]{2}{*}{$\begin{array}{l}\text { RP ajustada ** } \\
2003-2008\end{array}$} & \multirow[t]{2}{*}{ Valor de $p$} & \multirow[t]{2}{*}{$\begin{array}{c}\text { RP ajustada ** } \\
2003-2015\end{array}$} & \multirow[t]{2}{*}{ Valor de $p$} \\
\hline & $\%$ & IC95\% & $\%$ & IC95\% & $\%$ & IC95\% & & & & \\
\hline $\begin{array}{l}\text { Hipertensão } \\
\text { arterial } \\
\text { Medidas de } \\
\text { controle }\end{array}$ & 11,7 & $9,4-14,5$ & 15,8 & $13,9-17,9$ & 16,0 & $14,3-17,9$ & $1,25(0,98-1,59)$ & 0,064 & $1,27(1,03-1,60)$ & 0,043 \\
\hline Dieta alimentar & 25,6 & $18,4-34,5$ & 50,9 & $42,0-59,9$ & 38,3 & $31,7-45,4$ & $2,04(1,42-2,91)$ & $<0,001$ & $1,51(1,05-2,15)$ & 0,025 \\
\hline Atividade física & 9,2 & $4,4-18,3$ & 12,0 & $7,5-18,6$ & 8,8 & $5,9-13,0$ & $1,29(0,55-3,05)$ & 0,557 & $0,95(0,43-2,13)$ & 0,901 \\
\hline $\begin{array}{l}\text { Medicamento } \\
\text { oral }\end{array}$ & 75,6 & $65,6-83,4$ & 74,0 & $66,8-80,2$ & 76,1 & $70,9-80,6$ & $1,01(0,86-1,17)$ & 0,938 & $1,01(0,88-1,16)$ & 0,894 \\
\hline Não faz nada & 12,2 & $7,1-20,2$ & 8,6 & $5,1-14,2$ & 7,7 & $5,5-10,7$ & $0,65(0,31-1,36)$ & 0,248 & $0,62(0,33-1,16)$ & 0,138 \\
\hline
\end{tabular}

IC95\%: intervalo de 95\% de confiança; RP: razão de prevalência.

* Número de indivíduos na amostra não ponderada;

** RP ajustada por sexo e idade (categoria de referência: ISA-Capital 2003). 
Tabela 3

Prevalências e razões de prevalências ajustadas de diabetes mellitus e hipertensão arterial e suas medidas de controle em população idosa (60 anos e mais), segundo ano de realização dos inquéritos. ISA-Capital 2003, 2008 e 2015, São Paulo, Brasil.

\begin{tabular}{|c|c|c|c|c|c|c|c|c|c|c|}
\hline \multirow[t]{2}{*}{ Variáveis } & \multicolumn{2}{|c|}{$\begin{array}{c}\text { ISA-Capital } 2003 \\
\quad(n=872) *\end{array}$} & \multicolumn{2}{|c|}{$\begin{array}{l}\text { ISA-Capital } 2008 \\
\quad(n=924) *\end{array}$} & \multicolumn{2}{|c|}{$\begin{array}{c}\text { ISA-Capital } 2015 \\
\quad(n=1.019) *\end{array}$} & \multirow[t]{2}{*}{$\begin{array}{c}\text { RP ajustada ** } \\
2003-2008\end{array}$} & \multirow[t]{2}{*}{ Valor de p } & \multirow[t]{2}{*}{$\begin{array}{c}\text { RP ajustada** } \\
2003-2015\end{array}$} & \multirow[t]{2}{*}{ Valor de $p$} \\
\hline & $\%$ & IC95\% & $\%$ & IC95\% & $\%$ & IC95\% & & & & \\
\hline Diabetes mellitus & 17,6 & $14,9-20,6$ & 20,1 & $17,3-23,1$ & 22,5 & $20,0-25,2$ & $1,15(0,96-1,42)$ & 0,194 & $1,29(1,08-1,56)$ & 0,012 \\
\hline \multicolumn{11}{|l|}{ Medidas de controle } \\
\hline Dieta alimentar & 52,0 & $41,8-62,1$ & 61,0 & $52,6-68,8$ & 45,9 & $38,7-53,4$ & $1,19(0,94-1,51)$ & 0,154 & $0,90(0,68-1,17)$ & 0,438 \\
\hline Atividade física & 2 & $0,3-13,0$ & 13,4 & $7,7-22,3$ & 6,1 & $3,6-10,2$ & $6,91(0,89-53,3)$ & 0,064 & $3,03(0,38-24,0)$ & 0,293 \\
\hline Medicamento oral & 60,8 & $51,3-69,5$ & 71,8 & $65,2-77,7$ & 83,7 & $77,2-88,7$ & $1,19(1,00-1,40)$ & 0,050 & $1,38(1,17-1,63)$ & $<0,001$ \\
\hline Insulina & 15,1 & $9,3-23,5$ & 15,8 & $11,2-26,0$ & 19,8 & $14,7-26,2$ & $1,09(0,62-1,93)$ & 0,761 & $1,36(0,79-2,33)$ & 0,266 \\
\hline Não faz nada & 5,6 & $3,1-10,0$ & 3 & $1,3-6,4$ & 11,1 & $7,5-16,1$ & $0,54(0,20-1,47)$ & 0,230 & $2,02(0,96-4,25)$ & 0,062 \\
\hline Hipertensão arterial & 46,2 & $41,5-51,5$ & 52,0 & $47,7-56,3$ & 54,9 & $51,0-58,6$ & $1,12(0,98-1,29)$ & 0,094 & $1,19(1,05-1,39)$ & 0,008 \\
\hline \multicolumn{11}{|l|}{ Medidas de controle } \\
\hline Dieta alimentar & 33,4 & $27,7-39,7$ & 51,8 & $43,4-60,1$ & 39,9 & $34,3-45,8$ & $1,57(1,23-2,00)$ & $<0,001$ & $1,20(0,95-1,51)$ & 0,117 \\
\hline Atividade física & 5,4 & $3,1-9,3$ & 10,5 & $7,0-15,4$ & 8,3 & $5,8-11,7$ & $1,96(0,98-3,85)$ & 0,053 & $1,50(0,79-2,88)$ & 0,215 \\
\hline Medicamento oral & 87,1 & $81,9-91,0$ & 92,8 & $90,0-94,8$ & 88,5 & $84,5-91,6$ & $1,06(1,00-1,13)$ & 0,034 & $1,02(0,95-1,09)$ & 0,617 \\
\hline Não faz nada & 1,6 & $0,7-3,5$ & 0,2 & $0,1-1,1$ & 1,3 & $0,8-3,4$ & $0,31(0,10-1,01)$ & 0,066 & $1,03(0,34-3,09)$ & 0,963 \\
\hline
\end{tabular}

IC95\%: intervalo de 95\% de confiança; RP: razão de prevalência.

* Número de indivíduos na amostra não ponderada;

** RP ajustada por sexo e idade (categoria de referência: ISA-Capital 2003).

No período de estudo, observou-se aumento nas prevalências de diabetes e hipertensão para os dois domínios de idade selecionados. Um trabalho realizado com dados de 751 inquéritos de base populacional em 146 países, analisou a tendência de diabetes entre 1980 e 2014: nenhum país apresentou decréscimo significativo nas prevalências, sendo que o Brasil foi o quarto país com o maior número de pessoas vivendo com diabetes no ano de 201423 . Outros estudos que analisaram dados de inquéritos brasileiros, domiciliares 24 e telefônicos 25,26, também encontraram aumento nas prevalências de diabetes para todo o território nacional e para a cidade de São Paulo.

Com relação à hipertensão, análise sistemática utilizando dados de inquéritos de base populacional de 90 países encontrou aumento nas prevalências de hipertensão em países de baixa e média rendas 27. No Brasil, um estudo 28 que estimou a tendência temporal de hipertensão encontrou aumento nas prevalências no período pesquisado; da mesma maneira, o VIGITEL 26 também apresentou variação temporal significativa (2011-2016) para a doença.

O aumento nas prevalências das duas doenças pode ter influência de vários fatores: maior expectativa de vida e maior proporção de idosos na população, além de estilo de vida sedentário acompanhado de dieta rica em açúcares e gorduras, que resultam em aumento da obesidade 2,29. Entretanto, deve-se considerar a ampliação do acesso a serviços de saúde e, com isto, a um aumento de diagnósticos e tratamentos, resultado de políticas públicas implantadas no país 30 .

Desde a implantação do Sistema Único de Saúde (SUS) no início da década de 1990, e impulsionada pelo processo de descentralização do sistema de saúde, a atenção básica, principal porta de entrada, tem como objetivo proporcionar acesso universal e cuidado longitudinal, além de implementar ações de promoção da saúde e prevenção de doenças 31. Um dos grandes desafios da atenção básica é a atenção em saúde para doenças crônicas. Nesse cenário, o Ministério da Saúde instituiu diretrizes e metodologias para o manejo e cuidado de pessoas com doenças crônicas, e mais especificamente, para diabetes e hipertensão, uma vez que o controle destas doenças é, também, responsabilidade da atenção básica 32 .

Contudo, para que o controle seja efetivo são necessárias intervenções intersetoriais diversas, que incluam ações no âmbito do controle das doenças crônicas e prevenção de agravos relacionados, como previsto no Plano de Ações Estratégicas para DCNT no Brasil 2011-2022. Dentre as principais ações 
instituídas nos últimos anos, podemos citar: o Programa Academia da Saúde, que promove ações que estimulam a atividade física e práticas corporais; o Programa Nacional de Calçadas Saudáveis, que viabilizou a (re)construção e melhoria de locais destinados à prática de atividade física, como parques, pistas de caminhadas e ciclovias; a implementação do Guia Alimentar para a População Brasileira, que traz recomendações sobre alimentação como forma de promoção da saúde e melhoria da qualidade de vida; o acordo com a indústria de alimentos, uma parceria entre o Ministério da Saúde, o setor privado e a sociedade civil para reduzir sal e açúcar nos alimentos; a redução dos preços de alimentos saudáveis, redução de impostos e aumento de subsídios com o propósito de aumentar o consumo destes; a criação de ações de comunicação, com o intuito de promover modos de vida saudável em nível nacional por meio de programas de televisão e rádio; entre outros 1.

Apesar dos esforços citados, no presente estudo a medida autorreferida prática de atividade física para diabetes mellitus e hipertensão arterial não apresentou diferença significativa no período. A atividade física apresentou baixa prevalência de autorrelato, mesmo sendo considerada tratamento não medicamentoso das doenças em questão 33. Para a dieta alimentar, as prevalências de autorrelato foram mais elevadas entre as pessoas que referiram diabetes do que entre aquelas que referiram hipertensão, mas o aumento desta prática foi significativo apenas para o último grupo. A baixa adesão a tratamento não medicamentoso para diabetes mellitus e hipertensão arterial já foi relatada em outros trabalhos $34,35,36$.

Em um estudo 37 realizado com dados da PNS 2013 foram analisadas as recomendações e práticas de comportamentos saudáveis entre pessoas com diabetes mellitus e hipertensão arterial. Mais de $80 \%$ dos indivíduos que referiram ter diabetes mellitus e/ou hipertensão arterial relataram ter recebido recomendações sobre práticas de comportamentos saudáveis nos atendimentos de saúde. Porém, não foram encontrados efeitos significativos na adoção de algumas medidas de controle, como a prática de atividade física e o consumo recomendado de frutas e hortaliças.

Apesar do aumento encontrado para a prática dieta alimentar entre os indivíduos que referiram hipertensão, deve-se considerar diferenças entre os questionários utilizados. No ano de 2003, indagou-se sobre a prática de dieta sem sal e nos anos seguintes sobre a prática de dieta com redução de sal. Tal alteração pode ter influenciado no aumento do autorrelato nos anos 2008 e 2015 . O uso de medicamentos apresentou altas prevalências de autorrelato entre os hipertensos nos três anos de estudo e aumento significativo no período para os indivíduos que referiram diabetes. Esses resultados corroboram as iniciativas e estratégias das políticas de assistência farmacêutica no país 38,39 , que garantem acesso a um grupo de medicamentos disponibilizados na rede de atenção básica e priorizam o controle das DCNT 40. O tratamento medicamentoso, devidamente efetuado e aliado a mudanças no estilo de vida, promove o controle das doenças e a qualidade de vida de seus portadores 41 .

Dentre os eixos que fundamentam o Plano de DCNT 1, o cuidado integral visa à expansão da atenção farmacêutica e da distribuição gratuita de medicamentos para hipertensão arterial e diabetes mellitus. Desde 2011, por meio da campanha Saúde Não Tem Preço, medicamentos para hipertensão arterial e diabetes mellitus são ofertados gratuitamente nas farmácias próprias do programa ou em drogarias privadas que tenham convênio com o Ministério da Saúde 39,42. Estima-se que tal medida tenha proporcionado um aumento de $70 \%$ na distribuição de medicamentos para a população diabética e hipertensa 1.

O Município de São Paulo especificamente adotou estratégias de atenção farmacêutica, como o Programa Remédio em Casa, uma iniciativa pública de distribuição de medicamentos em domicílio. Desde 2005, o programa disponibiliza medicamentos para um período de 90 dias e garante atendimento à população com hipertensão arterial, diabetes mellitus e hipotireoidismo em acompanhamento nas unidades básicas de saúde 43,44 .

É importante ressaltar o uso de inquéritos de saúde como produtor de informações vitais para a análise da situação de saúde de determinada população. Os resultados gerados são essenciais para o monitoramento e vigilância de indicadores, podendo apoiar o planejamento e execução de estratégias em saúde. Ainda, cabe evidenciar, também, a importância de coletar dados em nível regional ou local, no intuito de explorar com precisão a situação de saúde dos indivíduos residentes em dada localidade.

Deve-se enfatizar os diferentes processos de estratificação dos inquéritos utilizados, que podem influenciar a estimação da variância e, com isto, gerar ganhos ou perdas de precisão nas estimativas médias. Todavia, no processo de junção dos bancos de dados foram consideradas as diferenças no pro- 
cesso de estratificação dos inquéritos. Isso permitiu a utilização de módulo de análises em programa estatístico que considera distintos aspectos dos planos amostrais de cada um dos inquéritos. Por fim, a aplicação de modelos de regressão de Poisson em um único banco de dados permitiu a estimação das diferenças nos períodos 2003-2008 e 2003-2015. Estudos que comparam estimativas de dados oriundos de um mesmo inquérito, em diferentes períodos do tempo, ainda são escassos.

Assim, os resultados deste trabalho revelaram um panorama preocupante do Município de São Paulo: aumento significativo de hipertensão arterial e diabetes mellitus nos dois grupos etários analisados e baixa adesão a hábitos reconhecidamente benéficos à saúde e integrantes do tratamento destas doenças. A adesão ao tratamento não medicamentoso é um processo complexo e está além das ações de promoção da saúde na atenção básica.

Estudos já apontaram a relação entre o conhecimento e a atitude de práticas saudáveis: as pessoas reconhecem a importância da adoção destas práticas, mas poucos mostram motivação concreta para adotá-las 37,45. É necessário considerar os determinantes da escolha do estilo de vida, que muito provavelmente tem relações arraigadas no prazer sentido ao praticar alguns hábitos que são considerados não saudáveis 34 . Por exemplo, uma rotina diária de trabalho cansativa impacta na realização da atividade física no tempo livre. Encadear mudanças nos padrões educativos de modo a perpetuar práticas saudáveis é um processo lento e heterogêneo.

Por fim, é indispensável o estímulo ao estilo de vida saudável e a promoção de seus benefícios por meio de um conjunto de ações intersetoriais, além da implementação de políticas que proporcionem, também, ambientes favoráveis a este estilo de vida. Objeto de uma série de compromissos globais, as DCNT ganharam prioridade no âmbito da saúde na última década. Entretanto, o enfrentamento dos desafios complexos que elas implicam, essencialmente, vai além do setor saúde. Articular e alinhar ações efetivas e integradas é imprescindível na redução e controle das DCNT.

\section{Colaboradores}

S. R. Stopa foi responsável pela concepção do projeto, análise e interpretação dos dados, redação do artigo, revisão crítica do conteúdo e aprovação da versão final. C. L. G. Cesar, M. B. A. Barros e M. Goldbaum foram responsáveis pela concepção, revisão crítica do conteúdo e aprovação da versão final. N. J. Segri foi responsável pela concepção da análise, revisão crítica do conteúdo e aprovação da versão final. M. C. G. P. Alves foi responsável pela revisão crítica do conteúdo e aprovação da versão final.

\section{Agradecimentos}

Ao Conselho Nacional de Desenvolvimento Científico e Tecnológico (CNPq; processo no 140483/2016-0). 


\section{Referências}

1. Departamento de Análise de Situação de Saúde, Secretaria de Vigilância em Saúde, Ministério da Saúde. Plano de ações estratégicas para o enfrentamento das doenças crônicas não transmissíveis (DCNT) no Brasil, 2011-2022. Brasília: Ministério da Saúde; 2011.

2. World Health Organization. Global status report on noncommunicable diseases 2014. Geneva: World Health Organization; 2014.

3. Abegunde DO, Mathers CD, Adam T, Ortegon $\mathrm{M}$, Strong K. The burden and costs of chronic diseases in low-income and middle-income countries. Lancet 2007; 370:1929-38.

4. World Health Organization. Country profiles: Brazil 2014. Geneva: World Health Organization; 2014.

5. Malta DC, Stopa SR, Szwarcwald CL, Gomes NL, Silva-Júnior JB, Reis AAC. A vigilância e o monitoramento das principais doenças crônicas não transmissíveis no Brasil - Pesquisa Nacional da Saúde, 2013. Rev Bras Epidemiol 2015; 18 Suppl 2:3-16.

6. Iser BPM, Stopa SR, Chueiri PS, Szwarcwald CL, Malta DC, Monteiro HOC, et al. Prevalência de diabetes autorreferido no Brasil: resultados da Pesquisa Nacional de Saúde 2013. Epidemiol Serv Saúde 2015; 24:305-14.

7. Andrade SSA, Stopa SR, Brito AS, Chueiri PS, Szwarcwald CL, Malta DC. Prevalência de hipertensão arterial autorreferida na população brasileira: análise da Pesquisa Nacional de Saúde 2013. Epidemiol Serv Saúde 2015; 24:297-304.

8. Instituto Brasileiro de Geografia e Estatística. Pesquisa Nacional de Saúde 2013: percepção do estado de saúde, estilos de vida e doenças crônicas - Brasil, Grandes Regiões e Unidades da Federação. Rio de Janeiro: Instituto Brasileiro de Geografia e Estatística; 2014.

9. Secretaria de Gestão Estratégica e Participativa, Secretaria de Vigilância em Saúde, Ministério da Saúde. Vigitel Brasil 2006: vigilância de fatores de risco e proteção para doenças crônicas por inquérito telefônico. Brasília: Ministério da Saúde; 2007. (Série G. Estatística e Informação em Saúde)

10. Departamento de Vigilância de Doenças e Agravos não Transmissíveis e Promoção da Saúde, Secretaria de Vigilância em Saúde, Ministério da Saúde. Vigitel Brasil 2015: vigilância de fatores de risco e proteção para doenças crônicas por inquérito telefônico. Estimativas sobre frequência e distribuição sociodemográfica de de fatores de risco e proteção para doenças crônicas nas capitais dos 26 estados brasileiros e no Distrito Federal em 2015. Brasília: Ministério da Saúde; 2016.

11. Sociedade Brasileira de Cardiologia. 7a Diretriz Brasileira de Hipertensão Arterial. Arq Bras Cardiol 2016; 107 Suppl 3:1-83.
12. Oliveira JEP, Vencio S, organizadores. Diretrizes da Sociedade Brasileira de Diabetes (20152016). São Paulo: A.C. Farmacêutica; 2016.

13. Departamento de Atenção Básica, Secretaria de Atenção à Saúde, Ministério da Saúde. Estratégias para o cuidado da pessoa com doença crônica. Hipertensão arterial. Brasília: Ministério da Saúde; 2013. (Cadernos de Atenção Básica, 37).

14. Departamento de Atenção Básica, Secretaria de Atenção à Saúde, Ministério da Saúde. Estratégias para o cuidado da pessoa com doença crônica. Diabetes mellitus. Brasília: Ministério da Saúde; 2013. (Cadernos de Atenção Básica, 36).

15. Barros MBA. Inquéritos domiciliares de saúde: potencialidades e desafios. Rev Bras Epidemiol 2008; 11 Suppl 1:6-19.

16. Malta DC, Leal MC, Costa MFL, Morais Neto OL. Inquéritos Nacionais de Saúde: experiência acumulada e proposta para o inquérito de saúde brasileiro. Rev Bras Epidemiol 2008; 11:159-67.

17. Instituto Brasileiro de Geografia e Estatística. Pesquisa Nacional por Amostra de Domicílios Contínua. https://www.ibge.gov.br/estatisti cas-novoportal/sociais/trabalho/17270-pnadcontinua.html $=\& \mathrm{t}=\mathrm{o}$-que-e $($ acessado em 07/ Mai/2018).

18. ISA-Capital. Inquérito de Saúde no Município de São Paulo. http://www.fsp.usp.br/isa-sp (acessado em 10/Mai/2018).

19 Secretaria Municipal de Saúde de São Paulo. Aspectos metodológicos e produção de análises. http://www.prefeitura.sp.gov.br/cidade/ secretarias/upload/saude/arquivos/publica coes/ISA_2015_MA.pdf (acessado em 10/ Mai/2018).

20. Lee S, Davis WW, Nguyen HA, McNeel TS, Brick JM, Flores-Cervantes I. Examining trends and averages using combined cross-sectional survey data from multiple years (CHIS Methodology Paper). http://www.chis.ucla. edu/pdf/paper_trends_averages.pdf (acessado em 14/Out/2017).

21. Viacava F. Informações em saúde: a importância dos inquéritos populacionais. Ciênc Saúde Coletiva 2002; 7:607-21.

22. Duncan BB, Chor D, Aquino EML, Bensenor IM, Mill JG, Schmidt MI, et al. Doenças crônicas não transmissíveis no Brasil: prioridade para enfrentamento e investigação. Rev Saúde Pública 2012; 46 Suppl:126-34.

23. NCD Risk Factor Collaboration. Worldwide trends in diabetes since 1980: a pooled analysis of 751 population-based studies with 4.4 million participants. Lancet 2016; 387:1513-30. 
24. Freitas LRS, Garcia LP. Evolução da prevalência do diabetes e deste associado à hipertensão arterial no Brasil: análise da Pesquisa Nacional por Amostra de Domicílios, 1998, 2003 e 2008. Epidemiol Serv Saúde 2012; 21:7-19.

25. Malta DC, Iser BPM, Andrade SSCA, Moura L, Oliveira TP, Bernal RTI. Tendência da prevalência do diabetes melito autorreferido em adultos nas capitais brasileiras, 2006 a 2012. Epidemiol Serv Saúde 2014; 23:753-60.

26. Departamento de Vigilância de Doenças e Agravos não Transmissíveis e Promoção da Saúde, Secretaria de Vigilância em Saúde, Ministério da Saúde. Vigitel Brasil 2016: vigilância de fatores deBrasil. Ministério da Saúde. Vigitel Brasil 2016: vigilância de fatores de risco e proteção para doenças crônicas por inquérito telefônico. Brasília: Ministério da Saúde; 2017.

27. Mills KT, Bundy JD, Kelly TN, Reed JE, Kearney PM, Reynolds K, et al. Global disparities of hypertension prevalence and control: a systematic analysis of population-based studies from 90 countries. Circulation 2016; 134:44150.

28. Lobo LAC, Canuto R, Dias-da-Costa JS, Pattussi MP. Tendência temporal da prevalência de hipertensão arterial sistêmica no Brasil. Cad Saúde Pública 2017; 33:e00035316.

29. Schmidt MI, Duncan BB, Hoffmann JF, Moura L, Malta DC, Carvalho RM. Prevalence of diabetes and hypertension based on self-reported morbidity survey, Brazil, 2006. Rev Saúde Pública 2009; 43 Suppl 2:74-82.

30. Barros MBA, Francisco PMSB, Zanchetta LM, Cesar CLG. Tendências das desigualdades sociais e demográficas na prevalência de doenças crônicas no Brasil, PNAD: 2003-2008. Ciênc Saúde Coletiva 2011; 16:3755-68.

31. Paim J, Travassos C, Almeida C, Bahia L, Macinko J. The Brazilian health system: history, advances, and challenges. Lancet 2011; 377:1778-97.

32. Ministério da Saúde. Estratégias para o cuidado da pessoa com doença crônica. Brasília: Ministério da Saúde; 2013. (Cadernos de Atenção Básica, 35).

33. Nelson ME, Rejeski WJ, Blair SN, Duncan PW, Judge JO, King AC, et al. Physical activity and public health in older adults: recommendation from the American College of Sports Medicine and the American Heart Association. Med Sci Sports Exerc 2007; 39:1435-45.

34. Boas LCGV, Foss M, Foss-Freitas MC, Torres HDC, Monteiro LZ, Pace AE. Adesão à dieta e ao exercício físico das pessoas com diabetes mellitus. Texto \& Contexto Enferm 2011; 20:272-9.
35. Girotto E, Andrade SM, Cabrera MAS, Matsuo T. Adesão ao tratamento farmacológico e não farmacológico e fatores associados na atenção primária da hipertensão arterial. Ciênc Saúde Coletiva 2013; 18:1763-72.

36. Lakerveld J, Verstrate L, Bot SD, Kroon A, Baan $\mathrm{CA}$, Brug J, et al. Environmental interventions in low SES neighbourhoods to promote healthy behaviour: enhancing and impeding factors. Eur J Public Health 2014; 24:390-5.

37. Szwarcwald CL, Souza-Júnior PRB, Damacena GN, Almeida WS, Malta DC, Stopa SR, et al Recomendações e práticas dos comportamentos saudáveis entre indivíduos com diagnóstico de hipertensão arterial e diabetes no Brasil: Pesquisa Nacional de Saúde (PNS), 2013. Rev Bras Epidemiol 2015; 18 Suppl 2:132-45.

38. Brasil. Lei no 10.858, de 13 de abril de 2004. Autoriza a Fundação Oswaldo Cruz - Fiocruz - a disponibilizar medicamentos, mediante ressarcimento, e dá outras providências. Diário Oficial da União 2004; 14 abr.

39. Ministério da Saúde. Portaria no 184 , de 3 de fevereiro de 2011. Dispõe sobre o Programa Farmácia Popular do Brasil. Diário Oficial da União 2011; 4 fev.

40. Oliveira LCF, Assis MMA, Barboni AR. Assistência farmacêutica no Sistema Único de Saúde: da Política Nacional de Medicamentos à atenção básica à saúde. Ciênc Saúde Coletiva 2010; 15 Suppl 3:3561-7.

41. Gontijo MF, Ribeiro AQ, Klein CH, Rozenfeld S, Acurcio FA. Uso de anti-hipertensivos e antidiabéticos por idosos: inquérito em Belo Horizonte, Minas Gerais, Brasil. Cad Saúde Pública 2012; 28:1337-46.

42. Silva RM, Caetano R. Programa "Farmácia Popular do Brasil": caracterização e evolução entre 2004-2012. Ciênc Saúde Coletiva 2015; 20:2943-56.

43. Coordenação da Atenção Básica, Secretaria Municipal de Saúde de São Paulo. Programa Remédio em Casa: orientações aos profissionais e serviços de saúde. São Paulo: Secretaria Municipal de Saúde; 2012.

44. Mansour SN, Monteiro CN, Luiz OC. Adesão ao tratamento farmacológico de pacientes hipertensos entre participantes do Programa Remédio em Casa. Epidemiol Serv Saúde 2016; 25:647-54.

45. Lima MT, Bucher JSNF, Lima JWO. A hipertensão arterial sob o olhar de uma população carente: estudo exploratório a partir dos conhecimentos, atitudes e práticas. Cad Saúde Pública 2004; 20:1079-87. 


\section{Abstract}

The objective was to compare the estimates for prevalence of diabetes, hypertension, and behavioral measures to control these diseases. Data were analyzed for the adult population from Health Surveys in the city of São Paulo, Brazil, in 2003, 2008, and 2015. Prevalence rates and 95\% confidence intervals (95\% CI) were calculated for the following: hypertension, diabetes, and practices to control these diseases (diet, physical activity, oral medication, insulin, nothing). Estimates were compared by age and sex-adjusted Poisson regression and analyzed according to the 20-59-years and 60-and-older age brackets. The data were presented comparing 2008 to 2003 and 2015 to 2003. Among persons 20 to 59 years of age, there was an increase in the prevalence rates for: hypertension in 2003-2015 (PR = 1.27; 95\%CI: 1.03-1.60) and diet for both periods $(2003-2008, P R=2.04$; 95\%CI: 1.42-2.91; and 2003-2015, $P R=1.51$; 95\%CI: 1.05-2.15). Among persons 60 years and older: diabetes $(P R=1.29 ; 95 \%$ CI: 1.08-1.56) and oral medication to control diabetes $(P R=1.38$; 95\%CI: 1.17-1.63), both in 2003-2015; hypertension in 2003-2015 (PR = 1.19; 95\%CI: 1.05-1.39); and diet and oral medication to control hypertension in 2003-2008 (PR = 1.20; 95\% CI: 0.95-1.51 and $P R=1.02 ; 95 \% \mathrm{CI}: 0.95-1.09$, respectively). The results are important for surveillance and monitoring of the target indicators and provide backing for planning health care activities in the city of São Paulo. Linking and aligning effective and integrated interventions is indispensable for reducing and controlling these chronic noncommunicable diseases.

Hypertension; Diabetes Mellitus; Chronic Disease; Epidemiological Monitoring; Health Surveys

\section{Resumen}

El objetivo fue comparar las estimativas de prevalencia de diabetes, hipertensión y las medidas de control para estas enfermedades. Se analizaron datos de población adulta, procedentes de encuestas de salud en el municipio de São Paulo, Brasil, de 2003, 2008 y 2015. Se estimaron las prevalencias y sus intervalos de 95\% de confianza (IC95\%) para las variables: hipertensión, diabetes y prácticas de control para estas enfermedades (dieta alimentaria, actividad física, medicamento oral, insulina, no hacer nada). Las estimativas se compararon por regresión de Poisson, ajustada por sexo y edad, y se analizaron según los dominios 20-59 y 60 años o más. Los datos se presentaron comparándose los años de 2008, en relación a 2003, y 2015 en relación a 2003. Entre las personas de 20-59 años, se observó un aumento en las prevalencias de hipertensión durante el periodo 2003-2015 $(R P=$ 1,27; IC95\%: 1,03-1,60) y dieta alimentaria para ambos periodos (2003-2008, $R P=2$,04; IC95\%: 1,42-2,91; y 2003-2015, $R P=1,51$; IC95\%: 1,052,15). Entre las personas con 60 años o más: diabetes (RP: 1,29; IC95\%: 1,08-1,56) y medicamento oral para controlar la diabetes $(R P=1,38$; IC95\%: 1,17-1,63), ambos durante el período 2003-2015; hipertensión durante el periodo 2003-2015 $(R P=$ 1,19; IC95\%: 1,05-1,39); además de dieta alimentaria y medicamento oral para controlar la hipertensión, durante el período 2003-2008 $(R P=1,20$; IC95\%: 0,95-1,51 y $R P=1$,02; IC95\%: 0,95-1,09, respectivamente). Los resultados son importantes para la vigilancia y monitoreo de los indicadores analizados, $y$ proporcionan apoyo a la planificación de acciones en salud en el municipio de São Paulo. Coordinar y alinear acciones efectivas e integradas es imprescindible para la reducción y control de esas enfermedades.

Hipertensión; Diabetes Mellitus; Enfermedad Crónica; Monitoreo Epidemiológico; Encuestas Epidemiológicas
Recebido em 14/Nov/2017

Versão final reapresentada em 30/Mai/2018

Aprovado em 14/Jun/2018 